\title{
IL-33 Enhanced the Proliferation and Constitutive Production of IL-13 and IL-5 by Fibrocytes
}

\author{
Hisako Hayashi, Akiko Kawakita, Shintaro Okazaki, \\ Hiroki Murai, Motoko Yasutomi, and Yusei Ohshima \\ Department of Pediatrics, Faculty of Medical Sciences, University of Fukui, 23-3 Shimoaizuki, Matsuoka, \\ Yoshida-gun, Fukui 910-1193, Japan \\ Correspondence should be addressed to Yusei Ohshima; yohshima@u-fukui.ac.jp
}

Received 11 February 2014; Accepted 26 March 2014; Published 13 April 2014

Academic Editor: Enrico Heffler

Copyright (c) 2014 Hisako Hayashi et al. This is an open access article distributed under the Creative Commons Attribution License, which permits unrestricted use, distribution, and reproduction in any medium, provided the original work is properly cited.

Interleukin-33 appears to play important roles in the induction of allergic airway inflammation. However, whether IL-33 is involved in airway remodeling remains unclear. Because fibrocytes contribute to tissue remodeling in the setting of chronic inflammation, we examined the effects of IL-33 on fibrocyte functions. Fibrocytes were generated in vitro from peripheral blood mononuclear cells by culturing in the presence of platelet derived growth factors and the cells were stimulated with IL-33. IL-33 enhanced cell proliferation, $\alpha$-SMA expression, and pro-MMP-9 activity by the fibrocytes without increasing endogenous transforming growth factor- $\beta 1$ production. Fibrocytes constitutively expressed IL-13 and IL-5, and their production was augmented by stimulation with IL-33. Dexamethasone inhibited the functions of fibrocytes, but IL-33 made fibrocytes slightly refractory to the inhibitory effect of dexamethasone in terms of IL-13 production. Montelukast suppressed IL-13 production by nonstimulated fibrocytes but not those stimulated by IL-33. These findings suggest that IL-33 is involved in the airway remodeling process through its modulation of fibrocyte function independent of antigen stimulation. IL-33 might partially reduce the therapeutic effects of glucocorticoid and cysteinyl leukotriene receptor antagonist on fibrocyte-mediated Th2 responses.

\section{Introduction}

Fibrocytes are unique bone marrow-derived mesenchymal progenitor cells that are characterized by expression of hematopoietic cell markers (CD34, CD45, and leukocyte specific protein-1) and stromal cell markers (collagen I and proly4-hydroxylase) [1-3]. Fibrocytes are presumably derived from a subpopulation of $\mathrm{CD} 14^{+}$peripheral blood monocytes [4]. Fibrocytes are involved in chronic inflammation, tissue repair, and fibrosis and might be a significant source of lung fibroblasts and myofibroblasts in response to lung injury and in remodeling of the airway wall $[1,5-8]$. They can differentiate into $\alpha$-smooth muscle actin $\left(\alpha\right.$-SMA) ${ }^{+}$myofibroblasts and contribute to extracellular matrix generation leading to sub- epithelial fibrosis, airway basement membrane thickness $[7,9]$. Recent data obtained from clinical settings suggest that the number of circulating fibrocytes might be a biomarker for disease progression in chronic lung diseases including asthma $[10,11]$.

Epithelial damage is one of the pathophysiological features observed in asthma. It is presumed that damage to the bronchial epithelium results in release of cytokines and growth factors that affect innate and adaptive immunity to allergen [12]. Because fibrocytes preferentially infiltrate the subepithelial zone in asthma, factors released from the bronchial epithelium may promote the airway recruitment and proliferation of fibrocytes and might modulate fibrocyte function $[7,9,13]$.

IL-33 is a member of the IL-1 cytokine family. It induces adoptive Th 2 immunity and signals through a complex that includes membrane-bound ST2 protein (ST2L) and IL-1 receptor accessory protein [14-16]. The IL-33/ST2L signaling pathway has been implicated in allergen-induced airway inflammation and hyperresponsiveness. IL-33 protein 
was elevated in bronchoalveolar lavage fluids of moderate asthmatics compared to mild and normal subjects. Moreover, increased airway epithelial IL-33 protein expression was found in severe asthma [17]. Recently, IL-33 has been shown to induce the production of Th 2 cytokines IL-5 and IL-13 from innate immune cells without antigen stimulation and thereby might contribute to allergic pulmonary inflammation [1820].

Although IL-33 supposedly contributes to airway remodeling by initiating Th2-mediated airway inflammation and by enhancing proliferation and differentiation of vascular endothelial cells [14], it is uncertain whether IL-33 exacerbate airway remodeling independent of the adaptive immune response. Therefore, in this study we examined the effects of IL-33 on the proliferation and function of fibrocytes. We show that IL-33 might play an important role in airway remodeling through innate immune responses.

\section{Materials and Methods}

2.1. Cell Culture. Peripheral blood was obtained from healthy volunteers with their written informed consent. The study was approved by the ethics committees of the University of Fukui. Peripheral blood mononuclear cells (PBMCs) $\left(1.5 \times 10^{7}\right)$ isolated by Ficoll-Paque Plus (GE Healthcare Biosciences, Piscataway, NJ) were suspended in two $\mathrm{mL}$ of RPMI1640 + 10\% fetal bovine serum (FBS) + penicillin and streptomycin (Invitrogen Co. Carlsbad, CA) and cultured in fibronectin-coated, six-well plates at $37^{\circ} \mathrm{C}$ with $5 \% \mathrm{CO}_{2}$ for $72 \mathrm{~h}$. Nonadherent cells were washed out over time with periodical changes of medium. To maximize the fibrocyte yield and the purity of adherent cells, $10 \mathrm{ng} / \mathrm{mL}$ of platelet derived growth factor-BB (PeproTec Inc., Rocky Hill, NJ) was added on day 7 , as described previously [21]. After 2 weeks of culture, fibrocytes were harvested and then washed twice. The purity of the in vitro generated fibrocytes was $95 \%$ or greater as determined by flow cytometry [21]. Cells $\left(1.2 \times 10^{5}\right.$ cells, $0.6 \times 10^{5}$ cells, and $0.3 \times 10^{5}$ cells) were cultured in FBS-free RPIM1640 in fibronectin-coated 12-, 24-, and 96well plates, respectively. Medium was replaced the next day with fresh FBS-free RPIM1640. The cells were stimulated with IL-33 (PeproTec Inc.) in the presence or absence of dexamethasone (Sigma-Aldrich), montelukast (Merck \& Co. Inc., Whitehouse Station, NJ), or anti-TGF- $\beta 1$ antibody (R\&D Systems Inc., Minneapolis, MN). After $72 \mathrm{~h}$ of stimulation, cell proliferation was assessed by a colorimetric cell viability assay or a BrdU incorporation assay using a Cell Counting Kit-8 (Dojindo, Kumamoto, Japan) or a Cell Proliferation Kit (GE Healthcare Life Sciences, Uppsala, Sweden), respectively. Assays were done in triplicate wells. Relative viable cell numbers were determined by dividing the absorbance at $450 \mathrm{~nm}$ of stimulated fibrocytes by that of nontreated control fibrocytes.

2.2. RNA Isolation and RT-PCR. After a $24 \mathrm{~h}$ culture with stimulation, total RNA was isolated using an RNeasy Mini Kit (QIAGEN K.K., Tokyo, Japan). First-strand cDNA was synthesized from $0.5 \mu \mathrm{g}$ of total RNA using the Superscript
II First Strand System (Invitrogen Co.). Polymerase chain reaction (PCR) amplification of the ST2L gene and the soluble splicing variant ST2L (sST2) gene was carried out using specific primers. The primer sequences for ST2L were as follows: CTGTCTGGCCCTGAATTTGC (sense) and AGCAGAGTGGCCTCAATCCA (antisense). The primer sequences for sST2L were as follows: CTGTCTGGCCCTGAATTTGS (sense) and TGGAACCACACTCCATTCTGC (antisense). PCR amplification of the housekeeping gene $\beta$-actin was performed for each sample for normalization between samples.

2.3. Flow Cytometric Analysis. Cells were pretreated with BD Cytofix/Cytoperm (BD Biosciences, San Diego, CA) and then incubated with anti- $\alpha$-SMA monoclonal antibody (mAb) (Sigma-Aldrich) or isotype control IgG, along with human IgG in $\mathrm{BD}$ Perm/Wash (BD Biosciences). After washing, FITC-conjugated $\mathrm{F}\left(\mathrm{ab}^{\prime}\right)_{2}$ of goat anti-rabbit IgG (Rockland, Gilbertsville, PA) was added. Specific mean fluorescence intensities $(\triangle \mathrm{MFI})$ were determined by subtracting the isotype-matched control antibody fluorescence.

2.4. Intracytoplasmic Cytokine Staining. In order to enhance intracellular cytokine staining signals by blocking protein transport processes, cells were cultured in the presence of monensin (GolgiStop, BD Biosciences) for $3 \mathrm{~h}$. The cells were fixed and permeabilized with $-20^{\circ} \mathrm{C} 100 \%$ ethanol and then blocked for one hour with phosphate-buffered saline- $0.05 \%$ Tween 20, containing normal human IgG. The cells were stained with PE-conjugated anti-IL-13 antibody (BD Biosciences) or isotype control IgG. The images were obtained using Olympus IX70 inverted microscopes (Olympus Co., Tokyo, Japan).

2.5. Gelatin Zymography. Culture supernatants were resolved by $7.5 \%$ SDS-PAGE in the presence of one $\mathrm{mg} / \mathrm{mL}$ gelatin. After electrophoresis, gels were washed for $30 \mathrm{~min}$ in $2.5 \%$ Triton-X-100 at room temperature to remove SDS. Gels were then washed for $30 \mathrm{~min}$ and incubated overnight at $37^{\circ} \mathrm{C}$ in reaction buffer $(50 \mathrm{mM}$ Tris- $\mathrm{HCl}, 0.2 \mathrm{M} \mathrm{NaCl}, 5 \mathrm{mM} \mathrm{CaCl}$, $0.02 \%$ Brij35, and $\mathrm{pH}$ 7.6). After staining with Coomassie Brilliant Blue R-250, gelatin-degrading activities of matrix metalloproteinases (MMPs) were identified as clear zones of lysis against a blue background. Gelatin zymography standard was obtained from Millipore. Photographs of the zymograms were scanned using a LAS-3000 mini (Fuji Film, Tokyo, Japan), and the intensity of the digitalized bands was analyzed by Multi Gauge Version 3.0 (Fuji Film).

2.6. Cytokine Measurements. IL-5 and IL-13 concentrations were determined by two-site sandwich ELISAs, using the protocols recommended by the antibody suppliers. Antibody pairs and standard recombinant cytokines for ELISA were purchased from Thermo Fischer Scientific Inc. (Rockford, IL). TGF- $\beta 1$ levels were determined with ELISA kits (Invitrogen Co.). 


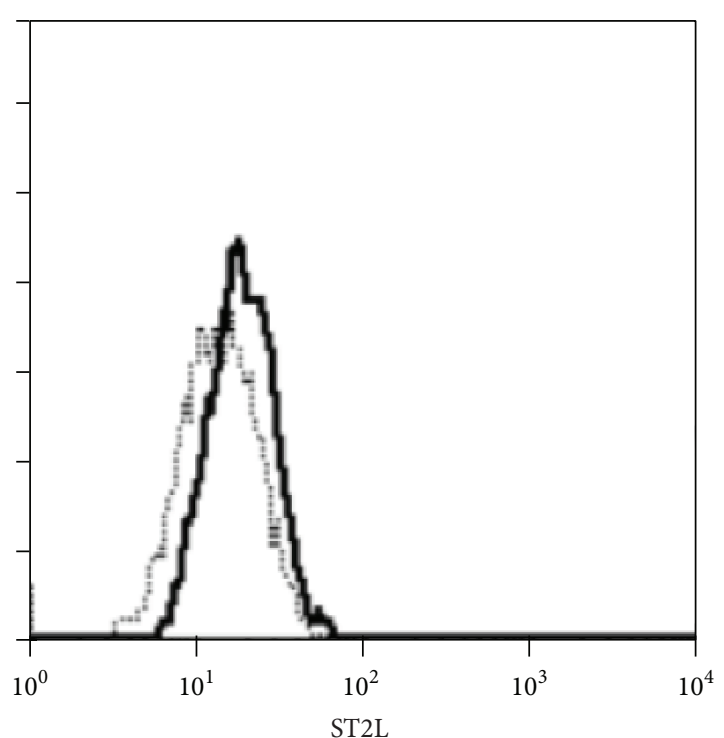

(a)

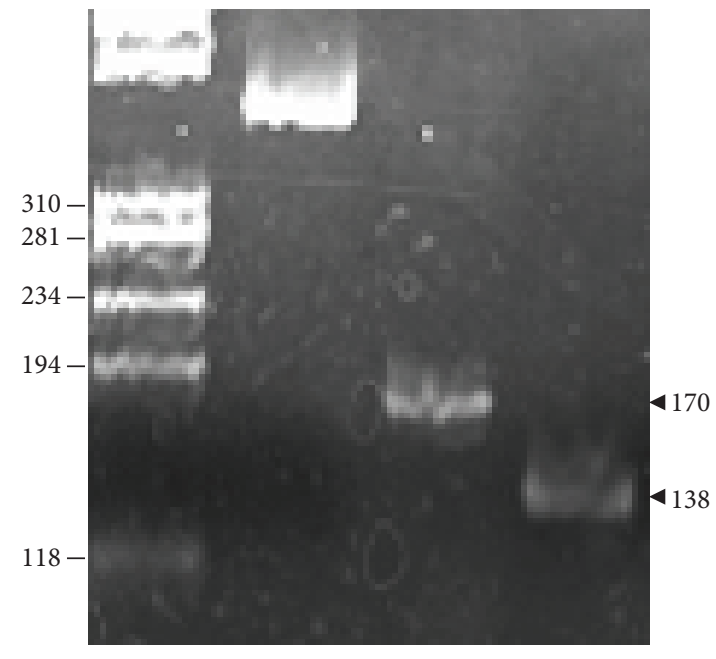

$\beta$-Actin ST2L sST2

(b)

FIgURE 1: ST2L and sST2 mRNA and protein expression by fibrocytes generated in vitro from PBMC. (a) Cell surface expression of ST2L protein on fibrocytes was detected by flow cytometry. Cells were stained with anti-ST2/IL-1R4 mAb (thick line) or isotype control IgG (thin line). (b) ST2L and sST2 mRNA expression was determined by RT- PCR.

2.7. Statistical Analysis. Comparisons of two groups used unpaired Student's $t$-tests, unless an $F$-test showed that the variances were significantly different. When variances were significantly different, Welch's test or paired Student's $t$-test was used. A $P$ value less than 0.05 denoted a statistically significant difference.

\section{Results}

3.1. Fibrocytes Generated from PBMCs Expressed ST2L and sST2. As previously reported [21], the fibrocytes generated from PBMCs expressed $\alpha$-SMA, calponin, collagen type I, and trace amounts of CD34, consistent with the typical phenotype of in vitro cultured fibrocytes, but did not express other cell lineage markers (i.e., dendritic cells; CD1 and CD83 and monocytes; CD14 and endothelial cells; von Willebrand factor and T cells; CD3 and B cells; CD19) (data not shown) [1].

It has been shown that circulating peripheral blood fibrocytes expressed ST2L [22]. Consistent with that finding, the fibrocytes generated from PBMC in vitro also expressed ST2L in mRNA and protein levels (Figure 1). The fibrocytes also expressed mRNA for sST2, another product of ST2 gene as a result of alternative splicing, which is presumed to acts as a decoy receptor to prevent IL-33 binding to and signaling through sT2L [14].

3.2. IL-33 Enhanced Fibrocyte Proliferation. Using a colorimetric assay, we observed that the relative number of viable fibrocytes generated in vitro was significantly increased in an IL-33 concentration-dependent manner, compared with nonstimulated control fibrocytes (Figure 2(a)). A BrdU incorporation assay confirmed that in vitro generated fibrocytes proliferated little, if any. However, IL-33 enhanced their proliferation at concentrations $\geq 10 \mathrm{ng} / \mathrm{mL}$ (Figure 2(b)). Since TGF- $\beta 1$ is profibrogenic growth factor and well known to induce fibrocyte differentiation [4], we asked whether the mitogenic effect of IL-33 depended on endogenously produced TGF- $\beta 1$. IL-33 did not increase TGF- $\beta 1$ production and neutralizing TGF- $\beta 1$ antibody did not suppress fibrocyte proliferation with or without IL-33 stimulation, indicating that the proliferation of in vitro generated fibrocytes was not attributable to endogenous TGF- $\beta 1$ (Figures 2(c) and 2(d)).

3.3. IL-33 Enhanced $\alpha$-SMA Expression and Pro-MMM-9 Activity. Fibrocytes are presumed to migrate into injured tissue by using MMPs and then transdifferentiate into myofibroblasts with the enhanced expression of $\alpha$-SMA [4, 23]. IL-33 increased the constitutive expression of $\alpha$-SMA protein, indicating that it induced transdifferentiation of fibrocytes (Figures 3(a) and 3(b)). Monocyte-derived fibrocytes are known to express various MMPs, including MMP2 and MMP-9 $[23,24]$. To evaluate the activity of MMP2 and MMP-9 produced by IL-33-stimulated fibrocytes, conditioned media were analyzed by gelatin zymography. As shown in Figures 3(c) and 3(d), gelatinolytic bands corresponding to MMP-9 and pro-MMP-9 were revealed, whereas those of MMP-2 and pro-MMP-2 were below detection. 


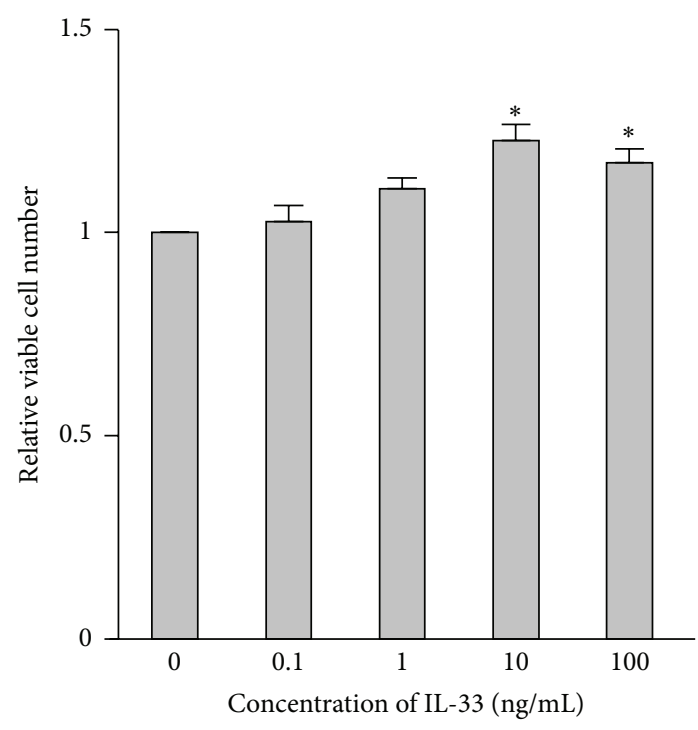

(a)

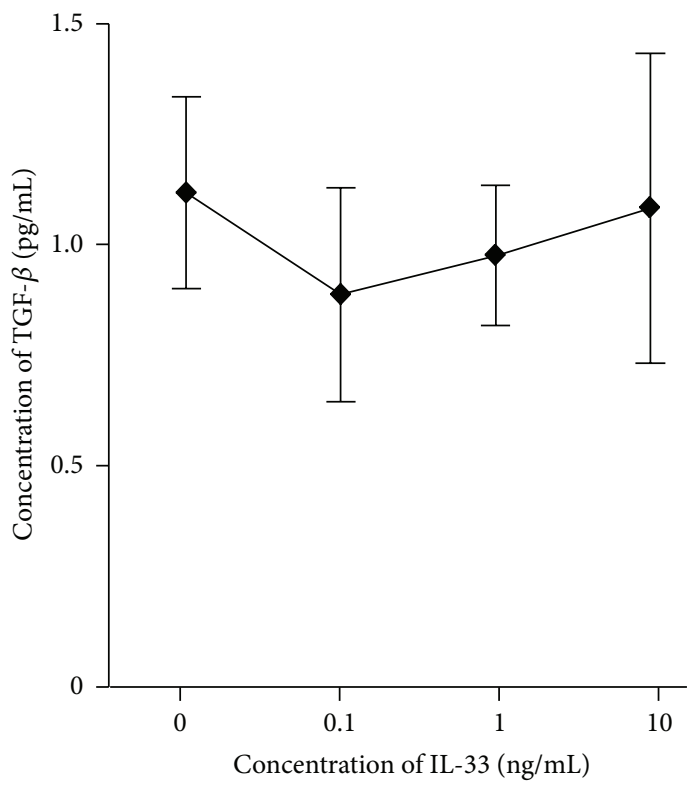

(c)

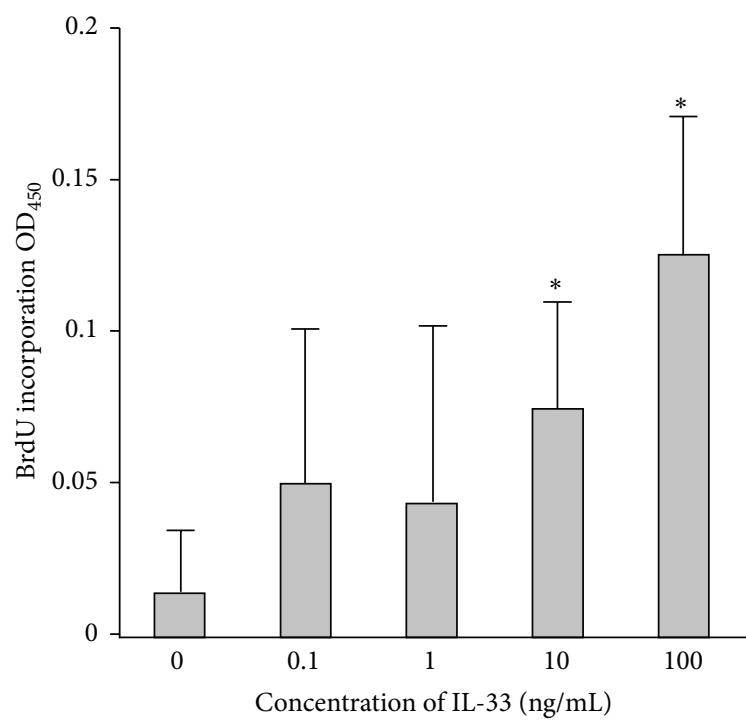

(b)

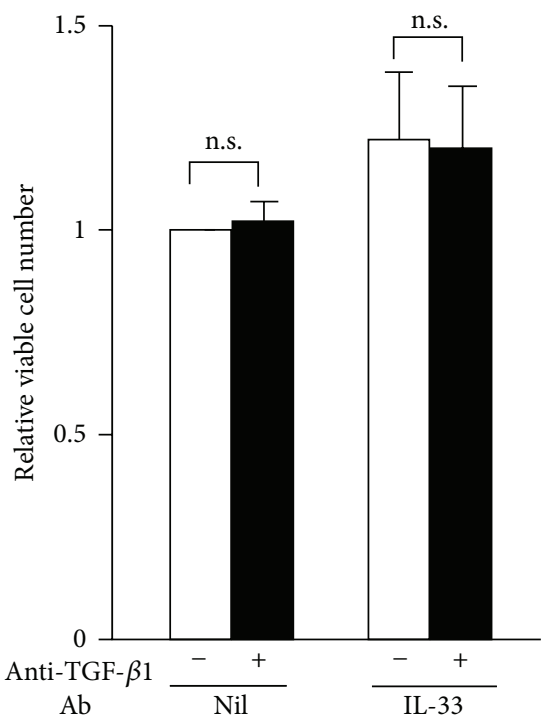

(d)

FIGURE 2: Effects of IL-33 on fibrocyte proliferation and endogenous TGF- $\beta 1$ production. Fibrocytes were cultured in the presence of various concentrations of IL-33. After $72 \mathrm{~h}$ of culture, relative viable cell number and DNA synthesis were determined by a colorimetric cell viability assay (a) and a BrdU incorporation assay (b), respectively. The concentration of TGF- $\beta 1$ in the supernatants was measured by ELISA (c). In (d), the relative proliferation of fibrocytes stimulated with or without $10 \mathrm{ng} / \mathrm{mL}$ IL-33 in the presence (closed bars) or absence (open bars) of $20 \mu \mathrm{g} / \mathrm{mL}$ anti-TGF- $\beta 1$ antibody was analyzed by a colorimetric assay. The data are expressed as means \pm SEM $(n=4)$. ${ }^{*} P<0.05$, n.s.: not significant.

The fibrocytes stimulated by IL-33 expressed increased gelatinolytic activity of pro-MMP-9 depending on the concentration of IL-33.

\subsection{IL-33 Enhanced Constitutive IL-13 and IL-5 Produc-} tion from Fibrocytes. We next examined whether fibrocytes were able to produce these type 2 cytokines without antigen stimulation. It is of note that fibrocytes constitutively produced IL-13 and IL-5, and $10 \mathrm{ng} / \mathrm{mL}$ IL-33 enhanced productions of these cytokines (Figures $4(\mathrm{a})$ and 4(b)). To further corroborate the constitutive production of IL13 from fibrocytes by themselves without either antigen or IL-33 stimulation, cells were cultured in the presence of monensin, an intracellular protein transport inhibitor, after which intracellular accumulation of IL-13 was assessed by immunofluorescence staining. As illustrated in Figure 4(c), immunofluorescence microscopy revealed the accumulation 


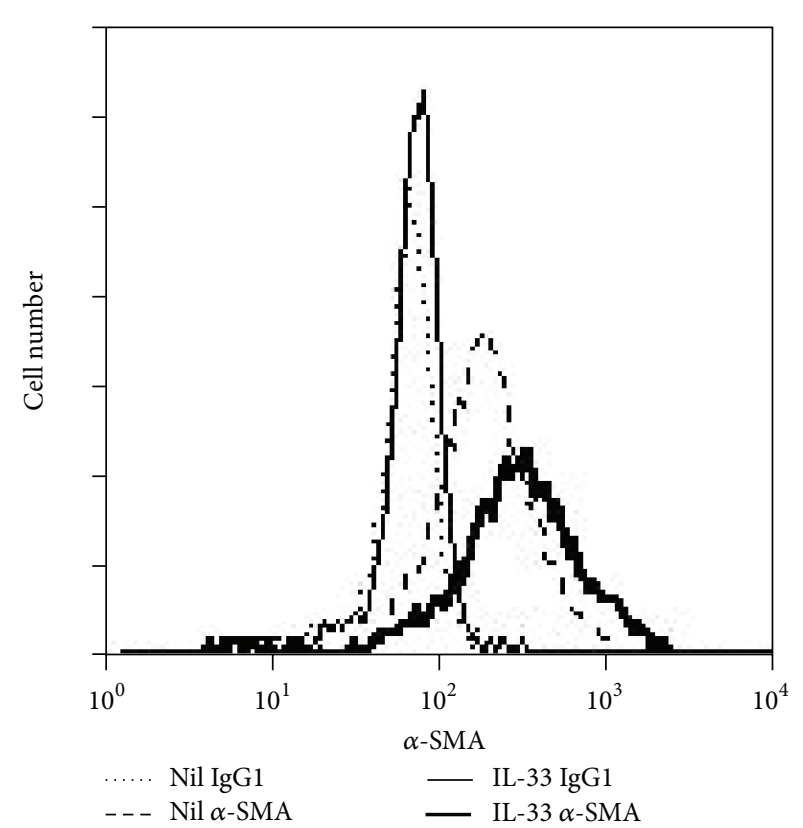

(a)

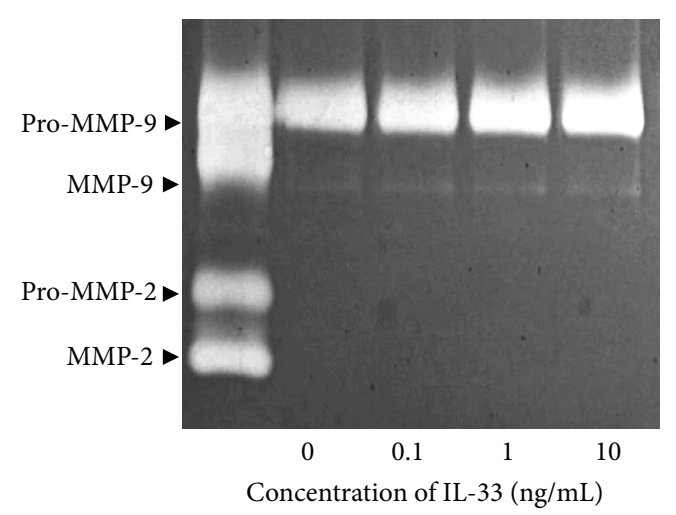

(c)

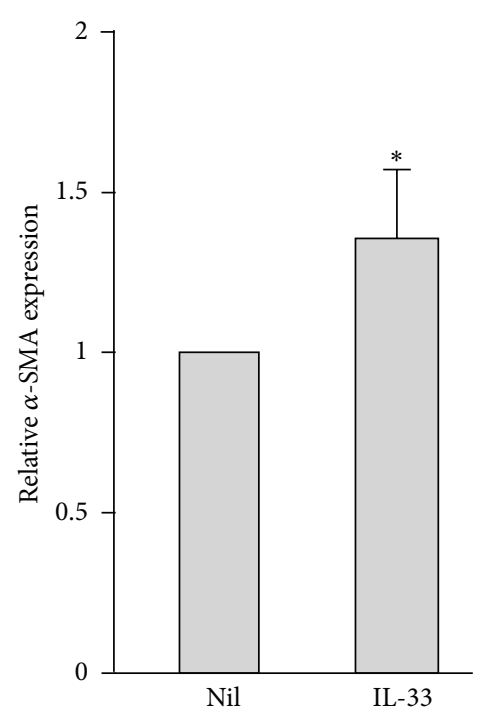

(b)

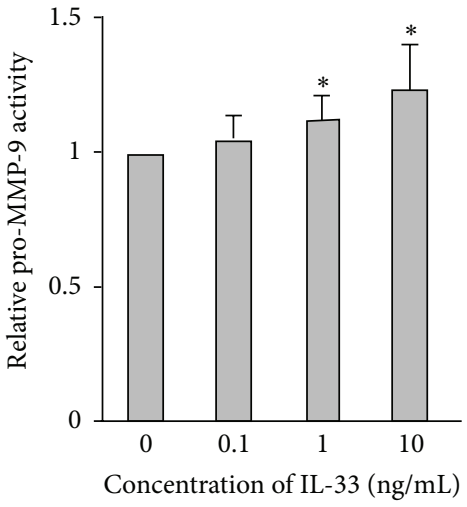

(d)

FIGURE 3: IL-33 enhanced $\alpha$-smooth muscle actin expression and pro-MMP-9 activity. Fibrocytes were cultured with (solid lines) or without (broken lines) $10 \mathrm{ng} / \mathrm{mL}$ IL-33 for $72 \mathrm{~h}$. Cells were stained with anti- $\alpha$-SMA mAb (thick line) or isotype control IgG (thin line) (a). Relative expression of $\alpha$-SMA with and without IL-33 was determined by flow cytometry (b). The gelatinase activities of MMP-2 and MMP-9 were analyzed by gelatin zymography (c) and its relative expression was determined by densitometry (d). Results are means \pm SEM ( $n=4)$. ${ }^{*} P<0.05$.

of constitutively produced IL-13 in spindle-shaped cells corresponding to fibrocytes.

\subsection{Dexamethasone Inhibited Proliferation, Cytokine Pro-} duction, and Pro-MMP9 Activity. The possible therapeutic effects of glucocorticoids on airway remodeling remain controversial. Dexamethasone (DEX) inhibited the proliferation of both nonstimulated and IL-33-stimulated fibrocytes in a dose dependent manner (Figure 5(a)). DEX did not affect the expression levels of ST2L and sST2 mRNA (data not shown), suggesting that DEX did not interfere with IL-33 signaling at the receptor level. IL-13 production by nonstimulated fibrocytes was suppressed by $\geq 1 \mu \mathrm{M}$ DEX, whereas that by IL-33-stimulated fibrocytes was suppressed by only $10 \mu \mathrm{M}$ DEX (Figure 5(b)). One $\mu \mathrm{M}$ DEX suppressed IL-13 production by nonstimulated and IL-33-stimulated fibrocytes $32.1 \pm 6.8$ and $8.0 \pm 7.2 \%$ (Mann-Whitney $U$ test; $P<0.05)$, respectively. Thus IL-33 stimulation might make fibrocytes relatively steroid resistant. $10 \mu \mathrm{M}$ DEX suppressed pro-MMP-9 activity expressed by both nonstimulated and IL33-stimulated fibrocytes (Figure 3(a)).

3.6. IL-33 Stimulation Made Fibrocytes Refractory to the Inhibitory Effects of Cysteinyl Leukotriene Receptor 1 Antagonists. Cysteinyl leukotrienes are key mediators of the airway remodeling process [25]. Both murine and human fibrocytes 


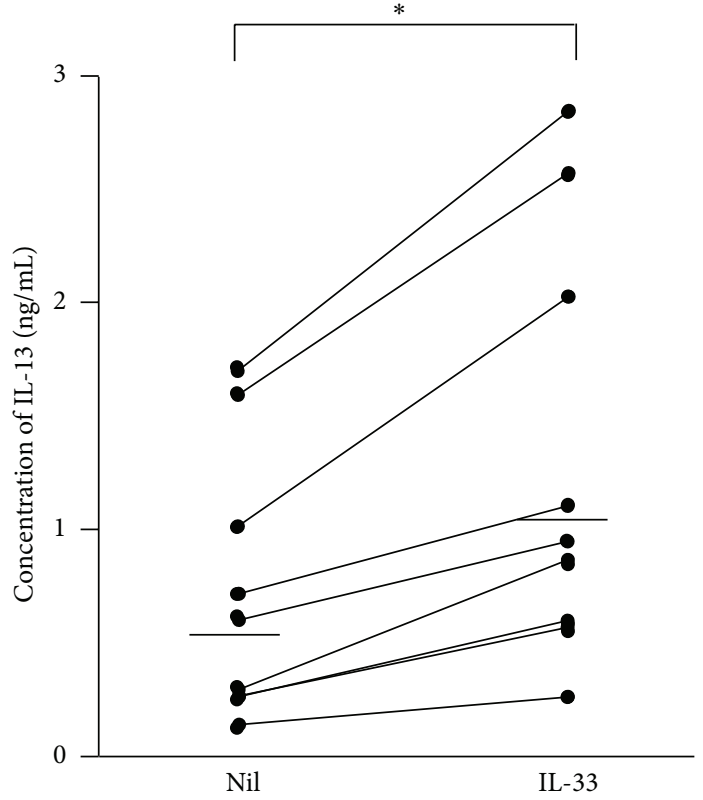

(a)

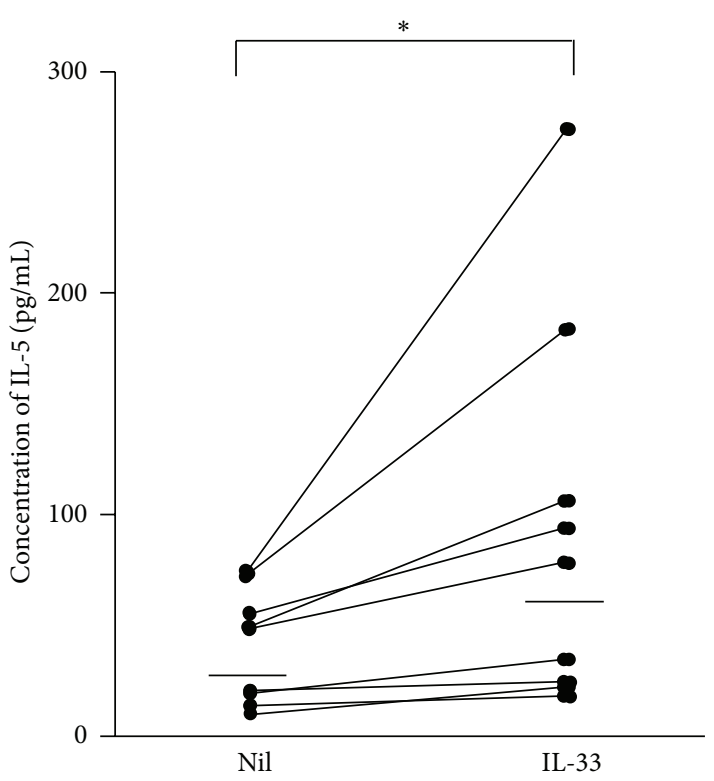

(b)

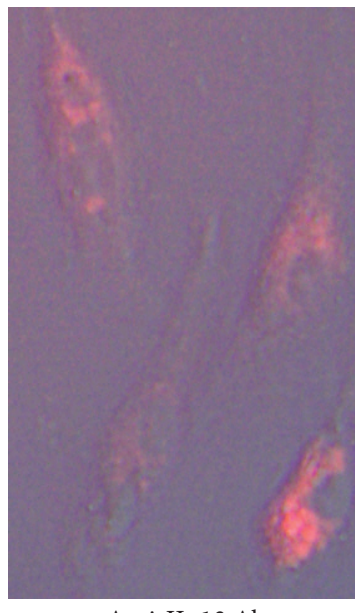

Anti-IL-13 Ab

Isotype

control Ig

(c)

FIgURE 4: IL-33 increased IL-13 and IL-5 production by fibrocytes. Fibrocytes were stimulated with $10 \mathrm{ng} / \mathrm{mL}$ of IL-33 for $72 \mathrm{~h}$. IL-13 (a) and IL-5 (b) levels in the supernatants were measured by ELISA. Horizontal thick bars represent the median of each group. Fibrocytes cultured in the presence of GolgiStop during the last three h of culture were stained with anti-IL-13 antibody (right panel) or isotype control IgG (left panel) (c). Intracytoplasmic IL-13 was detected by immunofluorescent microscopy. ${ }^{*} P<0.05$.

express both CysLT1 and CysLT2 [26]. CysLT1 antagonists inhibit exogenous $\mathrm{LTD}_{4}$-induced proliferation of murine fibrocytes. Since murine fibrocytes are capable of producing cysteinyl leukotrienes [27], we examined the effects of a CysLT1 antagonist, montelukast, on human fibrocyte function. Montelukast did not affect the expression levels of ST2L and SST2 mRNA (data not shown). As shown in Figure 6, montelukast did not inhibit proliferation but significantly suppressed constitutive IL-13 production and enhanced pro-MMP-9 activity expressed by human fibrocytes. The inhibitory effect of montelukast on IL-33-induced IL-13 production was not observed.

\section{Discussion}

In the present study, we have demonstrated that IL-33 induced the proliferation of fibrocytes generated in vitro and allowed these cells to differentiate into a myofibroblastic 


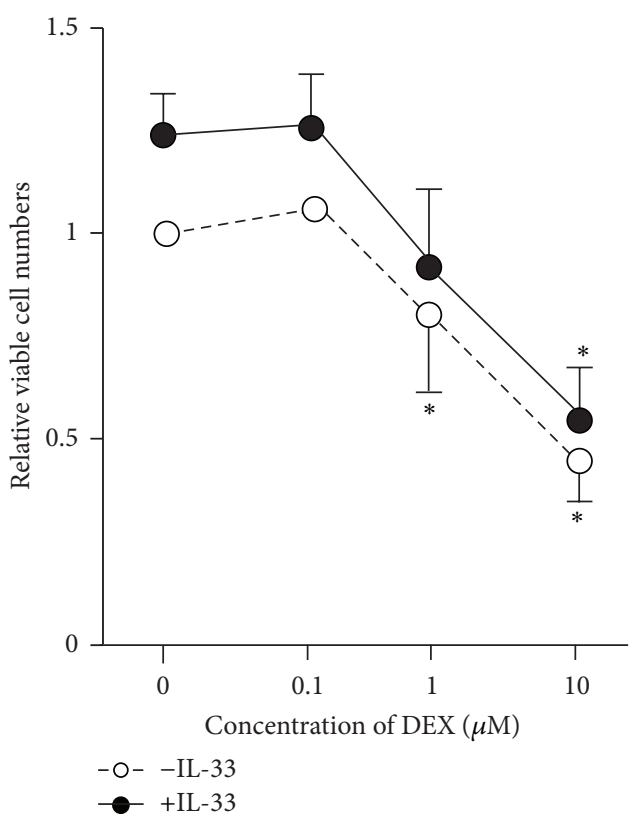

(a)

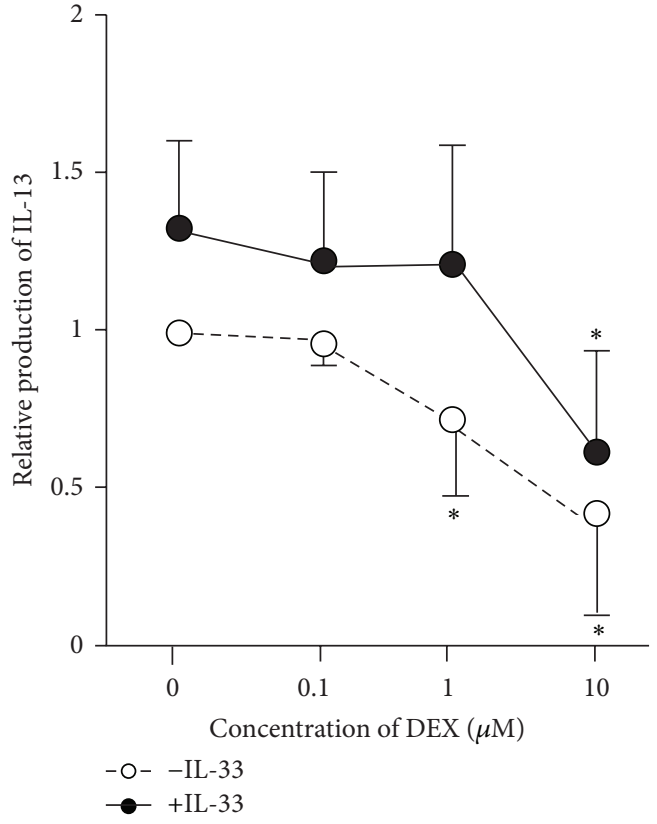

(b)

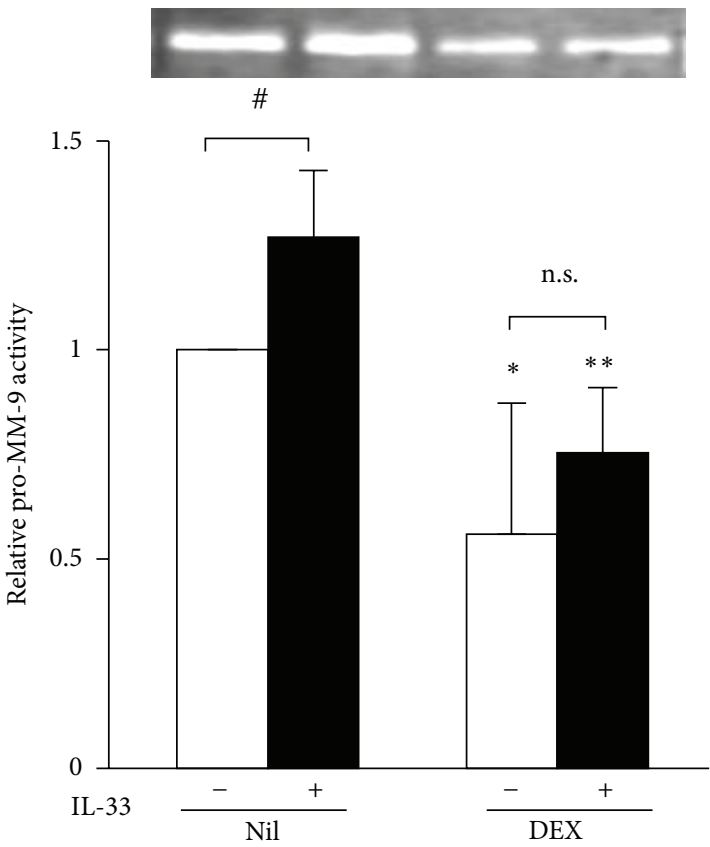

(c)

FIGURE 5: Effects of dexamethasone on cell proliferation, expression of IL-13, and pro-MMP-9 activity. Fibrocytes were cultured with (closed circles and bars) or without (open circles and bars) $10 \mathrm{ng} / \mathrm{mL} \mathrm{IL}-33$ for $72 \mathrm{~h}$ in the presence of various concentrations (a) and (b) or $10 \mu \mathrm{M}$ (c) dexamethasone. Relative proliferation (a), IL-13 production (b), and pro-MMP-9 activity (c) were analyzed by colorimetric cell viability assays, ELISA, and gelatin zymography, respectively. Results are means $\pm \operatorname{SEM}(n=6)$. ${ }^{*} P<0.05,{ }^{* *} P<0.01$ (compared with $0 \mu \mathrm{M}$ dexamethasone), ${ }^{\#} P<0.05$, n.s.: not significant.

phenotype. More intriguingly, the fibrocytes were able to constitutively produce type 2 cytokines, and IL-33 augmented the production of IL-13, a profibrogenic cytokine even without antigen stimulation. These data collectively suggest that IL-33 plays an important role in fibrocyte-mediated tissue remodeling.
Recently, Bianchetti et al. reported that IL-33 does not induce proliferation of circulating fibrocytes isolated from nonasthmatics but does enhance the proliferation of fibrocytes from patients with allergen-exacerbated asthma [22]. The difference in the responsiveness to IL-33 in terms of cell proliferation may be explained by the maturation stage 


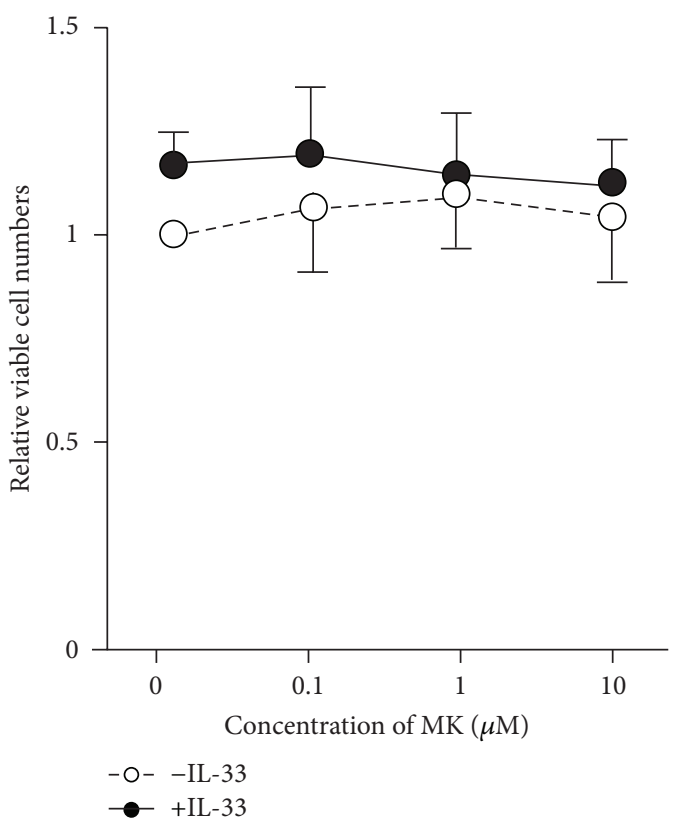

(a)

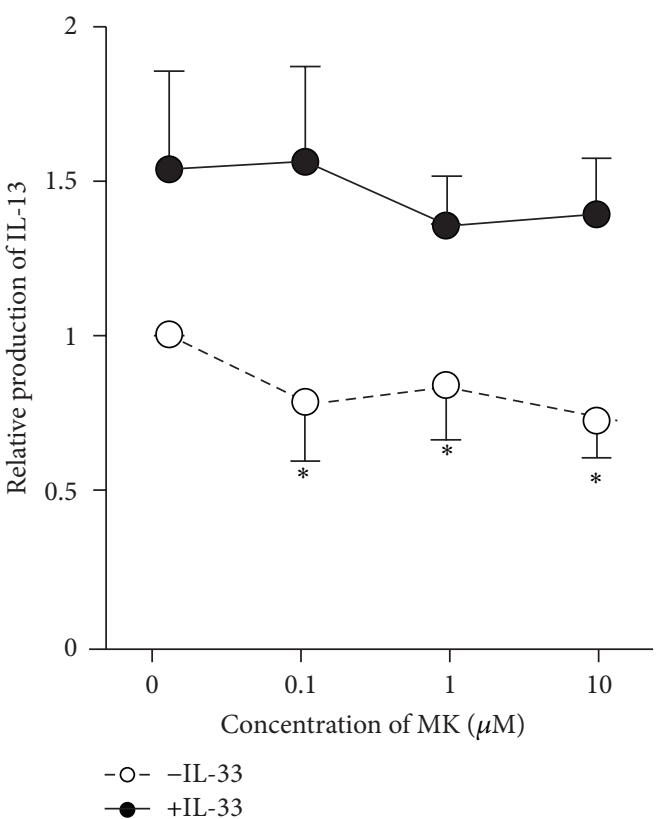

(b)

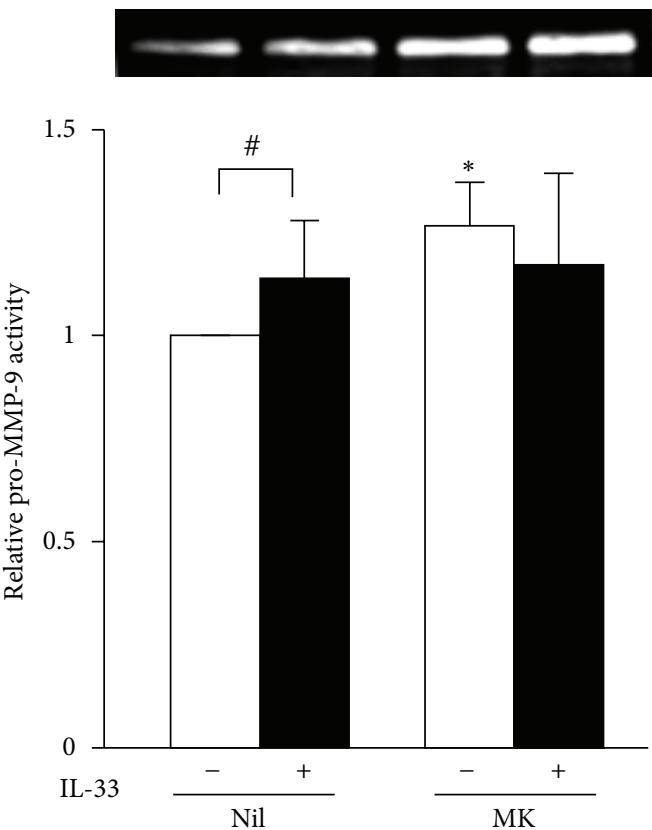

(c)

FIGURE 6: Effects of montelukast on cell proliferation, expression of IL-13, and pro-MMP-9 activity. Fibrocytes were cultured with (closed circles and bars) or without (open circles and bars) $10 \mathrm{ng} / \mathrm{mL}$ IL-33 for $72 \mathrm{~h}$ in the presence of various concentrations (a) and (b) or $10 \mu \mathrm{M}$ (c) montelukast (MK). Relative proliferation (a), IL-13 production (b), and pro-MMP-9 activity (c) were analyzed by colorimetric cell viability assays, ELISA, and gelatin zymography, respectively. Results are means $\pm \operatorname{SEM}(n=6) .{ }^{*} P<0.05$ (compared with $\left.0 \mu \mathrm{M} \mathrm{MK}\right)$, ${ }^{\#} P<0.05$, n.s.: not significant.

of fibrocytes. It has been reported that downregulation of CD34 and CD45RO expression occurs in parallel with an increased expression of $\alpha$-SMA as fibrocytes differentiate and mature $[7,26]$. Circulating fibrocytes robustly express CD34, whereas the fibrocytes generated in vitro express trace amounts of CD34 and large amounts of $\alpha$-SMA [21, 22]. Airway smooth muscle cell-derived PDGF has been shown to promote fibrocyte migration to smooth muscle bundles in vitro [28]. In this context, the fibrocytes generated in vitro by culturing in the presence of PDGF are maturer 
than circulating fibrocytes and might correspond to tissue resident fibrocytes recruited in the airway smooth muscle compartment in asthma.

Human $\mathrm{CD} 34^{+}$cells isolated from peripheral blood and cord blood express ST2L and respond to IL-33 ex vivo by secreting large quantities of Th2 cytokines [18]. Moreover, $\mathrm{CD} 34^{+}$cells producing IL-5 and IL-13 are detected in the sputum of individuals with allergic asthma. Around 25\% of fibroblasts-like cells in BAL fluid from asthma patients with basement membrane thickness express fibrocytes markers CD34 and $\alpha$-SMA [9]. Basement membrane thickness is correlated to the number of fibrocytes in tissue. Given that circulating $\mathrm{CD}_{3}{ }^{+}$cells are comprised of heterogeneous cell populations, fibrocytes could be important sources of IL13 and IL-5 in BAL fluid and airway tissues of asthmatics. Because IL-13 is an important cytokine involved in airway remodeling in asthma, IL-33 might augment airway remodeling through its enhancement of IL-13 production from fibrocytes without allergen stimulation $[29,30]$.

Bronchial epithelial cells of asthmatic patients express elevated levels of thymic stromal lymphopoietin (TSLP) as well as IL-33 [17, 30]. TSLP induces bronchial epithelial cell proliferation and increases injury repair through IL-13 [30]. TSLP expression of epithelial cells is enhanced by stimulation with IL-13 [31]. In this context, crosstalk between epithelial cells and fibrocytes via the IL-33/IL-13 axis might play an important role in airway remodeling in concert with TSLP.

Fibrocytes have been shown to produce and secrete MMP-2, MMP-7, MMP-8, and MMP-9 [23]. MMP-9 dissolves several extracellular matrix proteins and is implicated in the proteolysis of the basement membrane during the early invasion stage of angiogenesis as well as the migration of fibrocytes from the circulation to the tissues [23, 24]. Moreover, MMP-9 can activate latent TGF- $\beta$, a pleiotropic growth factor that directly induces a variety of responses associated with lung fibrosis and airway remodeling $[32,33]$. Therefore, IL-33 might facilitate angiogenesis and fibrosis in airway remodeling by enhancing pro-MMP-9 activity from fibrocytes.

We have previously reported that glucocorticoid suppresses cytokine production and upregulation of $\alpha$-SMA expression by activated fibrocytes [21]. Similarly, dexamethasone inhibited fibrocytes' proliferation and their expression of pro-MMP-9, which governs local accumulation of fibrocytes. In patients with chronic kidney diseases, it has been shown that the number of interstitial fibrocytes was significantly decreased by glucocorticoid therapy [34]. In this context, glucocorticoid may effectively prevent fibrocytes from accumulating at the sites of airway remodeling.

The inhibitory effect of montelukast on IL-13 production by nonstimulated fibrocytes was not due to cytotoxicity because IL-33-stimulated fibrocytes were refractory to the inhibitory effect of montelukast even at high concentrations. Endogenous cysteinyl leukotrienes released by fibrocytes might regulate constitutive IL-13 production in an autocrine fashion. Montelukast, but not DEX, was shown to reverse es-tablished features of airway remodeling, including subepithelial fibrosis in a chronic asthma mouse model [27]. Montelukast-mediated enhancement of pro-MMP-9 activity by fibrocytes might be involved in the reduction of subepithelial collagen deposition.

IL-33 and TNF- $\alpha$ expression in lung tissues from asthmatic subjects increases with the severity of asthma [35]. Dexamethasone fails to abolish TNF- $\alpha$-induced IL-33 upregulation in airway smooth muscle cells, which are a major source of IL-33 in asthma. We found that montelukast failed to suppress IL-13 production by IL-33-stimulated fibrocytes and that higher concentrations of dexamethasone were required to suppress it compared with nonstimulated fibrocytes, suggesting that IL-33 might partially reduce the therapeutic effects of glucocorticoid and cysteinyl leukotriene receptor antagonist on fibrocyte-mediated Th2 responses.

\section{Conclusions}

In conclusion, the IL-33-fibrocytes type 2 cytokine axis may account for the airway remodeling associated with allergic inflammation regardless of allergen-specific immune responses. IL-33 might play a pivotal role in the pathophysiology of refractory asthma characterized by persistent symptoms despite use of multiple regular asthma therapies, including inhaled corticosteroids and CysLTR antagonist.

\section{Conflict of Interests}

The authors declare that there is no conflict of interests regarding the publication of this paper.

\section{Acknowledgments}

The authors thank Ms. Mori and Ms. Toyooka for their excellent assistance in carrying out these studies. This work was supported in part by a Grant-in-Aid for Scientific Research from the Japan Society for the Promotion of Science (to Yusei Ohshima) and a Research Grant from Banyu Pharmaceutical Co. Ltd. (to Yusei Ohshima).

\section{References}

[1] E. L. Herzog and R. Bucala, "Fibrocytes in health and disease," Experimental Hematology, vol. 38, no. 7, pp. 548-556, 2010.

[2] R. A. Reilkoff, R. Bucala, and E. L. Herzog, "Fibrocytes: emerging effector cells in chronic inflammation," Nature Reviews Immunology, vol. 11, no. 6, pp. 427-435, 2011.

[3] H. Peng and E. L. Herzog, "Fibrocytes: emerging effector cells in chronic inflammation," Current Opinion in Pharmacology, vol. 12, no. 4, pp. 491-496, 2012.

[4] R. Abe, S. C. Donnelly, T. Peng, R. Bucala, and C. N. Metz, "Peripheral blood fibrocytes: differentiation pathway and migration to wound sites," Journal of Immunology, vol. 166, no. 12, pp. 7556-7562, 2001.

[5] M. W. Epperly, H. Guo, J. E. Gretton, and J. S. Greenberger, "Bone marrow origin of myofibroblasts in irradiation pulmonary fibrosis," American Journal of Respiratory Cell and Molecular Biology, vol. 29, no. 2, pp. 213-224, 2003. 
[6] R. J. Phillips, M. D. Burdick, K. Hong et al., "Circulating fibrocytes traffic to the lungs in response to CXCL12 and mediate fibrosis," Journal of Clinical Investigation, vol. 114, no. 3, pp. 438-446, 2004.

[7] M. Schmidt, G. Sun, M. A. Stacey, L. Mori, and S. Mattoli, "Identification of circulating fibrocytes as precursors of bronchial myofibroblasts in asthma," Journal of Immunology, vol. 171, no. 1, pp. 380-389, 2003.

[8] B. Mehrad, M. D. Burdick, D. A. Zisman, M. P. Keane, J. A. Belperio, and R. M. Strieter, "Circulating peripheral blood fibrocytes in human fibrotic interstitial lung disease," Biochemical and Biophysical Research Communications, vol. 353, no. 1, pp. 104-108, 2007.

[9] K. Nihlberg, K. Larsen, A. Hultgårdh-Nilsson, A. Malmström, L. Bjermer, and G. Westergren-Thorsson, "Tissue fibrocytes in patients with mild asthma: a possible link to thickness of reticular basement membrane?" Respiratory Research, vol. 7, p. 50, 2006.

[10] C. H. Wang, C. D. Huang, H. C. Lin et al., "Increased circulating fibrocytes in asthma with chronic airflow obstruction," American Journal of Respiratory and Critical Care Medicine, vol. 178, no. 6, pp. 583-591, 2008.

[11] A. Bellini, M. A. Marini, L. Bianchetti, M. Barczyk, M. Schmidt, and S. Mattoli, "Interleukin (IL)-4, IL-13, and IL-17A differentially affect the profibrotic and proinflammatory functions of fibrocytes from asthmatic patients," Mucosal Immunology, vol. 5, no. 2, pp. 140-149, 2012.

[12] B. N. Lambrecht and H. Hammad, "Asthma: the importance of dysregulated barrier immunity," European Journal of Immunology, vol. 43, no. 12, pp. 3125-3137, 2013.

[13] C. H. Wang, C. D. Huang, H. C. Lin et al., "Increased activation of fibrocytes in patients with chronic obstructive asthma through an epidermal growth factor receptor-dependent pathway," Journal of Allergy and Clinical Immunology, vol. 129, no. 5, pp. 1367-1376, 2012.

[14] T. Ohno, H. Morita, K. Arae et al., "Interleukin-33 in allergy," Allergy, vol. 67, no. 10, pp. 1203-1214, 2012.

[15] A. S. Mirchandani, R. J. Salmond, and F. Y. Liew, "Interleukin-33 and the function of innate lymphoid cells," Trends in Immunology, vol. 33, no. 8, pp. 389-396, 2012.

[16] R. J. Salmond, A. S. Mirchandani, A. G. Besnard et al., "IL33 induces innate lymphoid cell-mediated airway inflammation by activating mammalian target of rapamycin," The Journal of Allergy and Clinical Immunology, vol. 130, no. 5, pp. 1159-1166, 2012.

[17] D. Préfontaine, J. Nadigel, F. Chouiali et al., "Increased IL-33 expression by epithelial cells in bronchial asthma," Journal of Allergy and Clinical Immunology, vol. 125, no. 3, pp. 752-754, 2010.

[18] Z. Allakhverdi, M. R. Comeau, D. E. Smith et al., "CD34+ hemopoietic progenitor cells are potent effectors of allergic inflammation," Journal of Allergy and Clinical Immunology, vol. 123, no. 2, pp. 472-478, 2009.

[19] E. Hams and P. G. Fallon, "Innate type 2 cells and asthma," Current Opinion in Pharmacology, vol. 12, no. 4, pp. 503-509, 2012.

[20] J. A. Walker and A. McKenzie, "Innate lymphoid cells in the airways," European Journal of Immunology, vol. 42, no. 6, pp. 13681374, 2012.

[21] H. Hayashi, A. Kawakita, S. Okazaki et al., "IL-17A/F modulates fibrocyte functions in cooperation with CD40-mediated signaling," Inflammation, vol. 36, no. 4, pp. 830-838, 2013.
[22] L. Bianchetti, M. A. Marini, M. Isgro et al., "IL-33 promotes the migration and proliferation of circulating fibrocytes from patients with allergen-exacerbated asthma," Biochemical and Biophysical Research Communications, vol. 426, no. 1, pp. 116121, 2012.

[23] C. García-de-Alba, C. Becerril, V. Ruiz et al., "Expression of matrix metalloproteases by fibrocytes: possible role in migration and homing," American Journal of Respiratory and Critical Care Medicine, vol. 182, no. 9, pp. 1144-1152, 2010.

[24] I. Hartlapp, A. B. E. Riichiro, R. W. Saeed et al., "Fibrocytes induce an angiogenic phenotype in cultured endothelial cells and promote angiogenesis in vivo," FASEB Journal, vol. 15, no. 12, pp. 2215-2224, 2001.

[25] T. S. Hallstrand and W. R. Henderson Jr., "An update on the role of leukotrienes in asthma," Current Opinion in Allergy and Clinical Immunology, vol. 10, no. 1, pp. 60-66, 2010.

[26] R. Bucala, "Review series-inflammation \& fibrosis. Fibrocytes and fibrosis," QJM, vol. 105, no. 6, pp. 505-508, 2012.

[27] K. M. Vannella, T. R. McMillan, R. P. Charbeneau et al., "Cysteinyl leukotrienes are autocrine and paracrine regulators of fibrocyte function," Journal of Immunology, vol. 179, no. 11, pp. 7883-7890, 2007.

[28] R. Saunders, S. Siddiqui, D. Kaur et al., "Fibrocyte localization to the airway smooth muscle is a feature of asthma," Journal of Allergy and Clinical Immunology, vol. 123, no. 2, pp. 376-384, 2009.

[29] J. L. Ingram and M. Kraft, "IL-13 in asthma and allergic disease: asthma phenotypes and targeted therapies," The Journal of Allergy and Clinical Immunology, vol. 130, no. 4, pp. 829-842, 2012.

[30] A. Semlali, E. Jacques, L. Koussih, A. S. Gounni, and J. Chakir, "Thymic stromal lymphopoietin-induced human asthmatic airway epithelial cell proliferation through an IL-13-dependent pathway," Journal of Allergy and Clinical Immunology, vol. 125, no. 4, pp. 844-850, 2010.

[31] A. Kato, S. Favoreto Jr., P. C. Avila, and R. P. Schleimer, “TLR3and Th2 cytokine-dependent production of thymic stromal lymphopoietin in human airway epithelial cells," Journal of Immunology, vol. 179, no. 2, pp. 1080-1087, 2007.

[32] Q. Yu and I. Stamenkovic, "Cell surface-localized matrix metalloproteinase-9 proteolytically activates TGF- $\beta$ and promotes tumor invasion and angiogenesis," Genes and Development, vol. 14, no. 2, pp. 163-176, 2000.

[33] L. A. Murray, Q. Chen, M. S. Kramer et al., "TGF-beta driven lung fibrosis is macrophage dependent and blocked by Serum amyloid P," International Journal of Biochemistry and Cell Biology, vol. 43, no. 1, pp. 154-162, 2011.

[34] N. Sakai, K. Furuichi, Y. Shinozaki et al., "Fibrocytes are involved in the pathogenesis of human chronic kidney disease," Human Pathology, vol. 41, no. 5, pp. 672-678, 2010.

[35] D. Préfontaine, S. Lajoie-Kadoch, S. Foley et al., "Increased expression of IL-33 in severe asthma: evidence of expression by airway smooth muscle cells," Journal of Immunology, vol. 183, no. 8, pp. 5094-5103, 2009. 


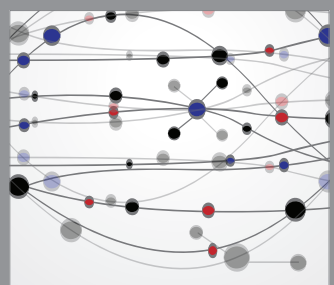

The Scientific World Journal
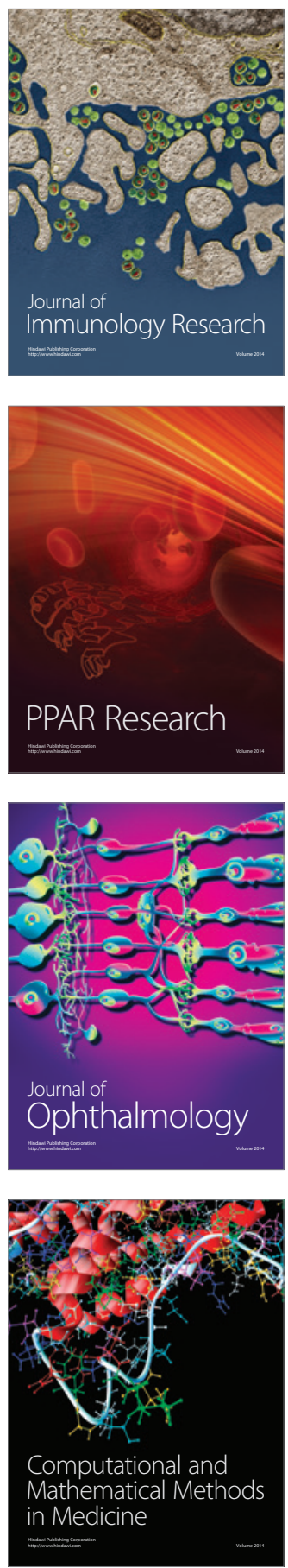

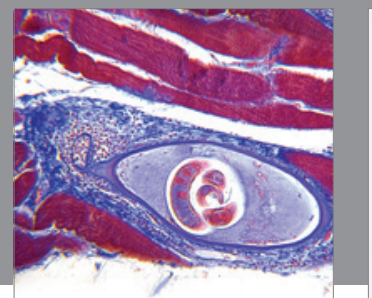

Gastroenterology

Research and Practice
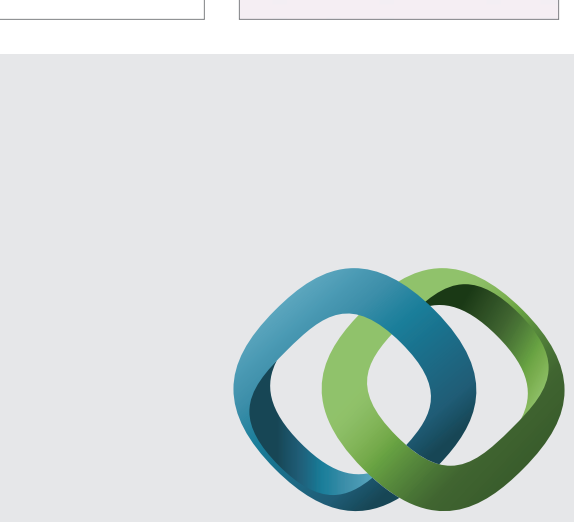

\section{Hindawi}

Submit your manuscripts at

http://www.hindawi.com
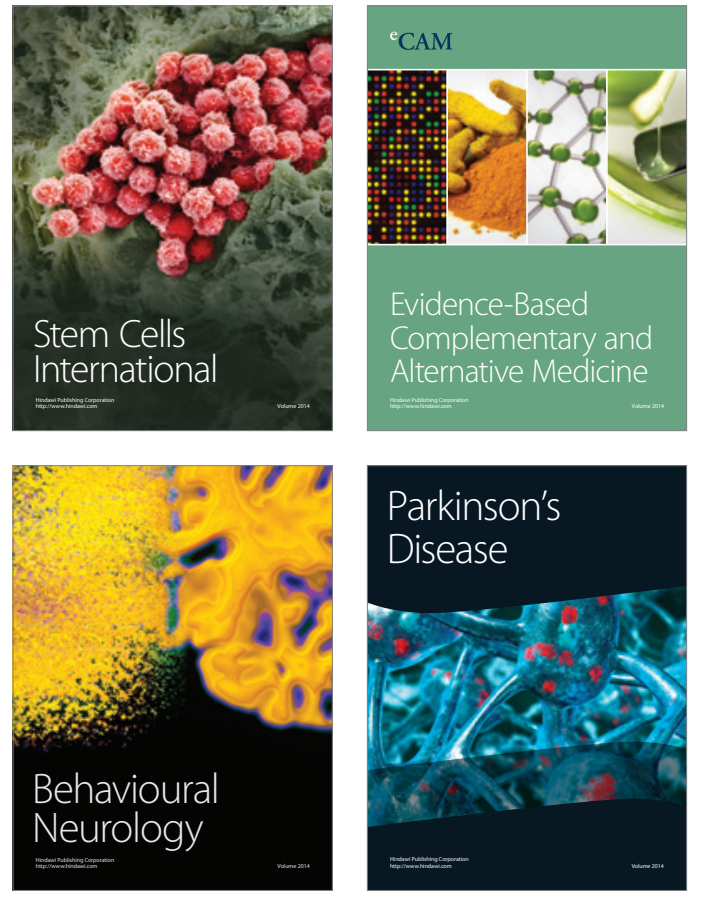
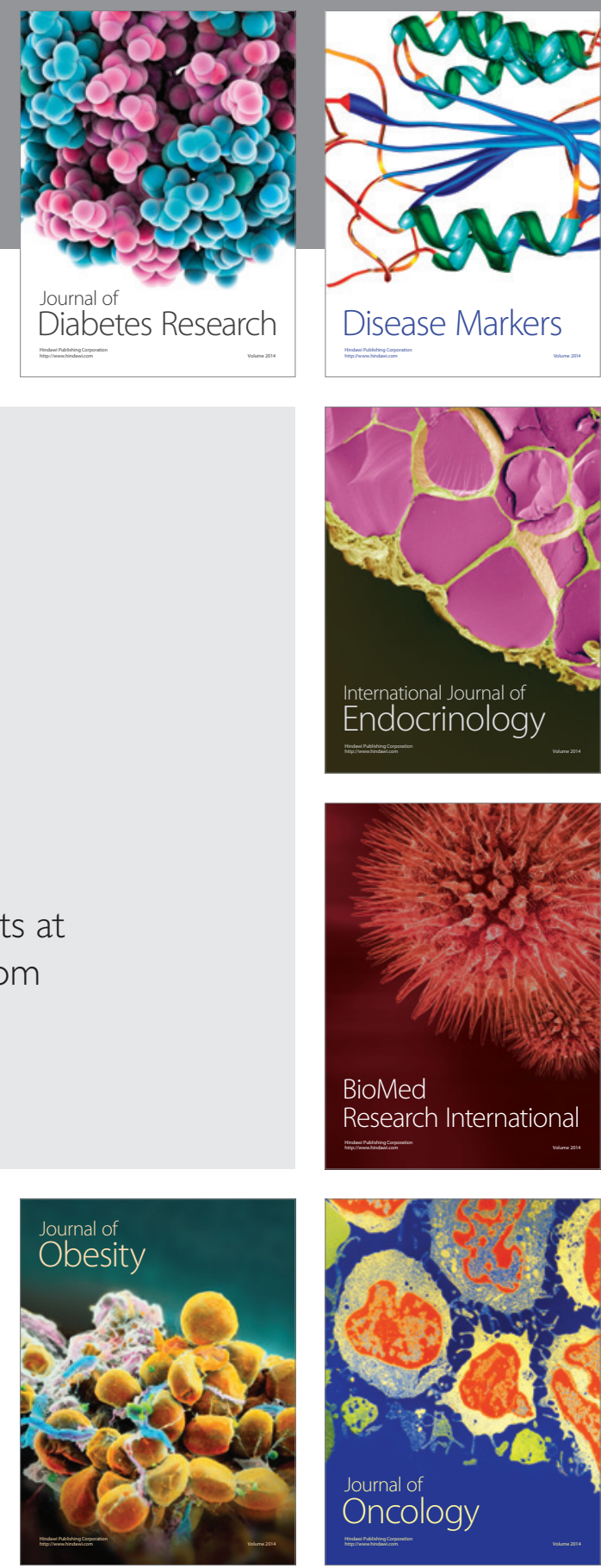

Disease Markers
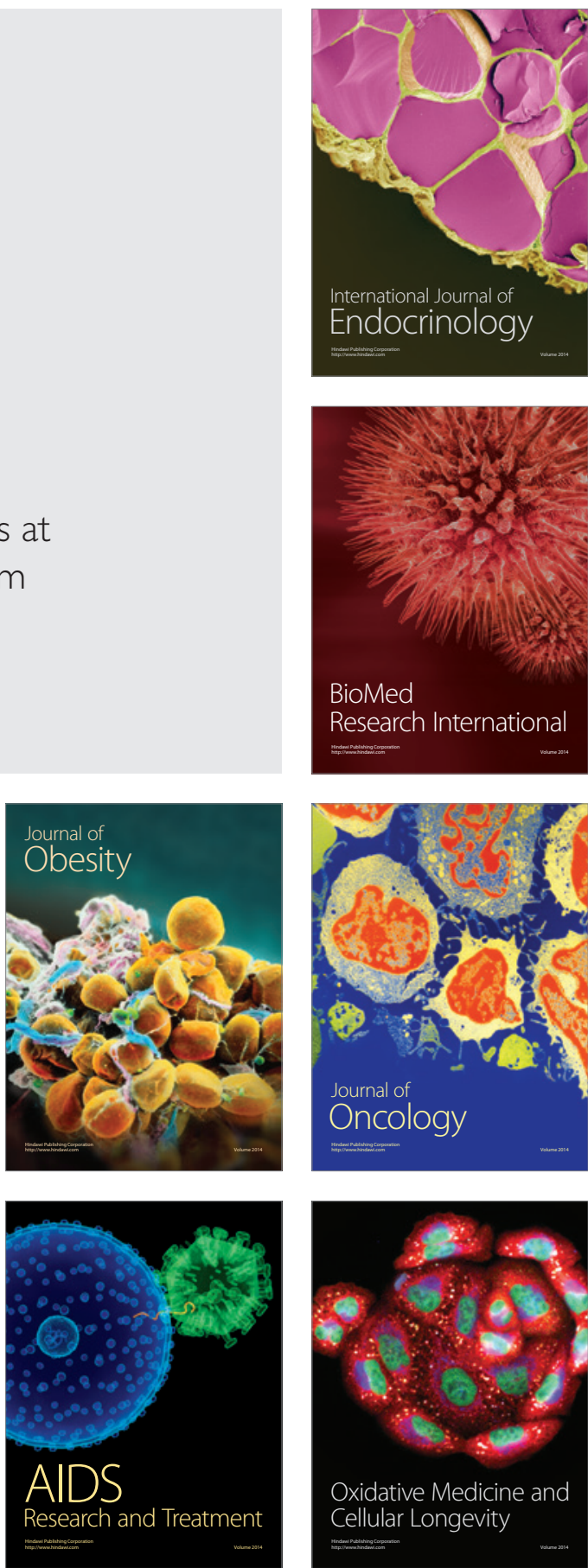